\title{
Primary Splenic Melanoma Associated with Small Intestinal Serosal Implants Causing Intussusception - A Case Report
}

\author{
Omar Hamdy ${ }^{1 *}$, Gehad A Saleh², Sara Raafat ${ }^{3}$, Mohammad Zuhdy ${ }^{1}$ and Abdelhadi M Shebl ${ }^{3}$
}

*Corresponding author:

Omar Hamdy, MD

Surgical Oncology Unit

Oncology Center Mansoura University

(OCMU), Mansoura, Egypt

Tel: +201003526752

E-mail: omarhamdy87@gmail.com
'Surgical Oncology Unit, Oncology Center Mansoura University (OCMU), Mansoura, Egypt ${ }^{2}$ Radiology Department, Faculty of Medicine, Mansoura University, Mansoura, Egypt

${ }^{3}$ Pathology Department, Faculty of Medicine, Mansoura University, Mansoura, Egypt

\section{ABSTRACT}

Introduction: Melanoma is a highly aggressive tumor that carries a poor prognosis. There are several reported cases of secondary splenic melanoma but there is only one reported case of primary splenic melanoma yet.

Case presentation: A 35-year-old male, complaining from left lumbar pain. Abdominal ultrasound revealed a mass at the lower pole of the spleen measuring 10x7 $77 \mathrm{~cm}$. Computed Tomography (CT) described a necrotic focal lesion involving its lower pole measuring $11 \mathrm{x}$ $9 \mathrm{~cm}$. US-guided fine needle aspiration (FNA) was suggestive of lymphoma, but tissue biopsy was recommended. So, the patient underwent open diagnostic splenectomy which revealed the diagnosis of malignant melanoma. Only two weeks after being discharged from the hospital, he was re-admitted by acute intestinal obstruction for which he underwent exploration which revealed an ileo-ileal intussusception near the ileocecal junction with multiple pigmented malignant nodules on the wall of the small intestine. Resection of the involved small intestinal part including intussusception was done. Pathological examination revealed malignant melanoma infiltrating the whole intestinal wall thickness from outside (confirming its metastatic nature) and infiltration of the mesenteric LN by the same tumor tissue. The patient died 6 months later after being diagnosed with brain metastasis.

Conclusion: Primary splenic melanoma is extremely rare with only one reported case in literature. This report is not only unique because of the rarity of the primary splenic melanoma but the intussusception caused by melanomatous serosal deposits is extremely rare as well.

Key words: melanoma, spleen, intussusception, primary

\section{INTRODUCTION}

Melanoma is a highly aggressive tumor. It is a melanocytic tumor that is not limited to the skin only but occurs in small intestinal mucosa and eyes. Despite being less common than other types of skin cancer such as squamous cell carcinoma and basal cell carcinoma, it carries the worst prognosis and it is the cause of the majority of skin cancer-related mortality. There are several reported cases of secondary splenic melanoma with improved prognosis after surgical resection in case of being solitary metastasis $(1,2)$. There is only one reported case of primary splenic melanoma yet (3). 


\section{CASE PRESENTATION}

The patient was a 35-year-old male, farmer, with no relevant medical or surgical history. He was presented with a complaint of left lumbar pain. Clinical examination revealed a mildly enlarged spleen with neither palpable peripheral lymph nodes nor other significant clinical findings.

Ultrasonographic (US) examination of the abdomen revealed a well-defined isoechoic soft tissue mass at the lower pole of the spleen measuring $10 \times 7 \times 7 \mathrm{~cm}$ and showing cystic degeneration and moderately increased vascularity. Post-contrast multidetector Computed Tomography (CT) described an enlarged spleen with a necrotic focal lesion involving its lower pole measuring $11 \times 9 \mathrm{~cm}$ (fig. 1). It described also thickened edematous small intestinal loops with a segment of intussusception seen at the left iliac fossa. All laboratory findings were within normal values apart from shooting Lactate Dehydrogenase (LDH) value which was $2115 \mathrm{U} / \mathrm{L}$.

US-guided fine needle aspiration (FNA) from the splenic focal lesion was done. Microscopic examination of the prepared smears revealed diffuse infiltration as well as groups of atypical large cells showing hyperchromasia, increased nucleo-cytoplasmic (N/C) ratio and a moderate degree of atypia and pleomorphism. Some tumor cells showed vacuolization. This picture was suggestive of lymphoma, however, tissue biopsy was recommended for confirmation of diagnosis.

So, the patient underwent a diagnostic splenectomy with an uneventful postoperative course. Microscopic examination of the splenectomy specimen revealed infiltration by a malignant tumoral proliferation arranged as sheets and groups of pleomorphic polyhedral to spindle cells showing high N/C ratio, vesicular nuclei, and prominent nucleoli. Areas of fibrous septations, hemorrhage, and necrosis, brownish pigmentations were detected as well. Immunohistochemical studies revealed the positivity of tumoral cells for Melan A, HMB45, S-100 and negativity for $\mathrm{CD} 3, \mathrm{CD} 20, \mathrm{CD} 5, \mathrm{BCl} 2$, synaptophysin, chromogranin, CD68, CD1a, CD31, CD34, and Pan CK. This led to the diagnosis of malignant melanoma (fig. 2).

Only two weeks after being discharged from the hospital, the patient was re-admitted after being presented with acute intestinal obstruction for which he underwent conservative management for five days. CT examination revealed markedly dilated small intestinal loops on top of an ileo-ileal intussusception, necrotic mesenteric lymph nodes (LNs) and enhancing small bowel serosal surface implants (fig. 3). Exploration revealed dilated small intestinal loops with an ileo-ileal intussusception near the ileocecal junction with multiple pigmented malignant nodules on the wall of the small intestine. Minute nodules upon the rectum and descending colon and multiple LNs at the root of mesentery were detected as well. Resection of the involved small intestinal segment including the intussusception was done with primary anastomosis as well as excision of one of the mesenteric LNs. Pathological examination revealed malignant melanoma infiltrating the whole intestinal wall thickness from outside (confirming its metastatic nature) and infiltration of the mesenteric $\mathrm{LN}$ by the same tumor tissue. This was confirmed by the positivity of tumor cells to both HMB45 and S100. The patient was discharged after regaining full oral feeding and normal bowel habits.

Unfortunately, the first postoperative brain CT showed a well-defined intra-axial enhanced space occupying lesion in the left frontal region. It measured about $1.5 \mathrm{~cm}$ in diameter and was surrounded by a moderate degree of vasogenic edema. Another similar smaller lesion was seen in the right posterior parietal region. Both lesions were described to be metastatic. Also, multiple enlarged left para-aortic LNs were described, and the largest one was $4.5 \mathrm{~cm}$ in diameter. The patient received immediately palliative whole-brain
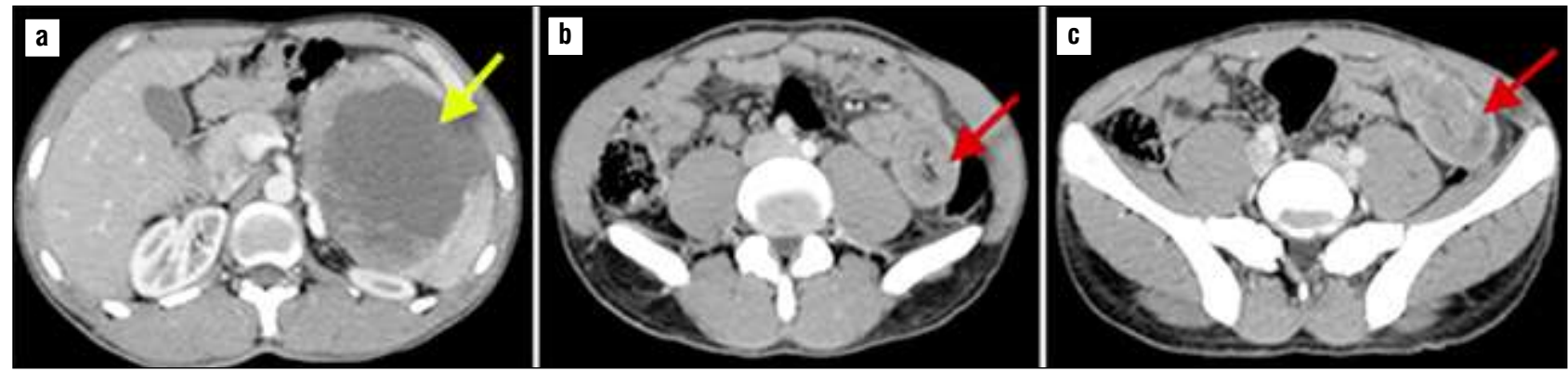

Figure 1 - Contrast-enhanced axial CT images revealed enlarged spleen with a large heterogeneously enhancing necrotic focal lesion (yellow arrow), no extra-capsular extension. Non obstructing small bowel intussusception at the left iliac region of typical target-like \& sausage-shaped mass (red arrows in B \& C respectively). 


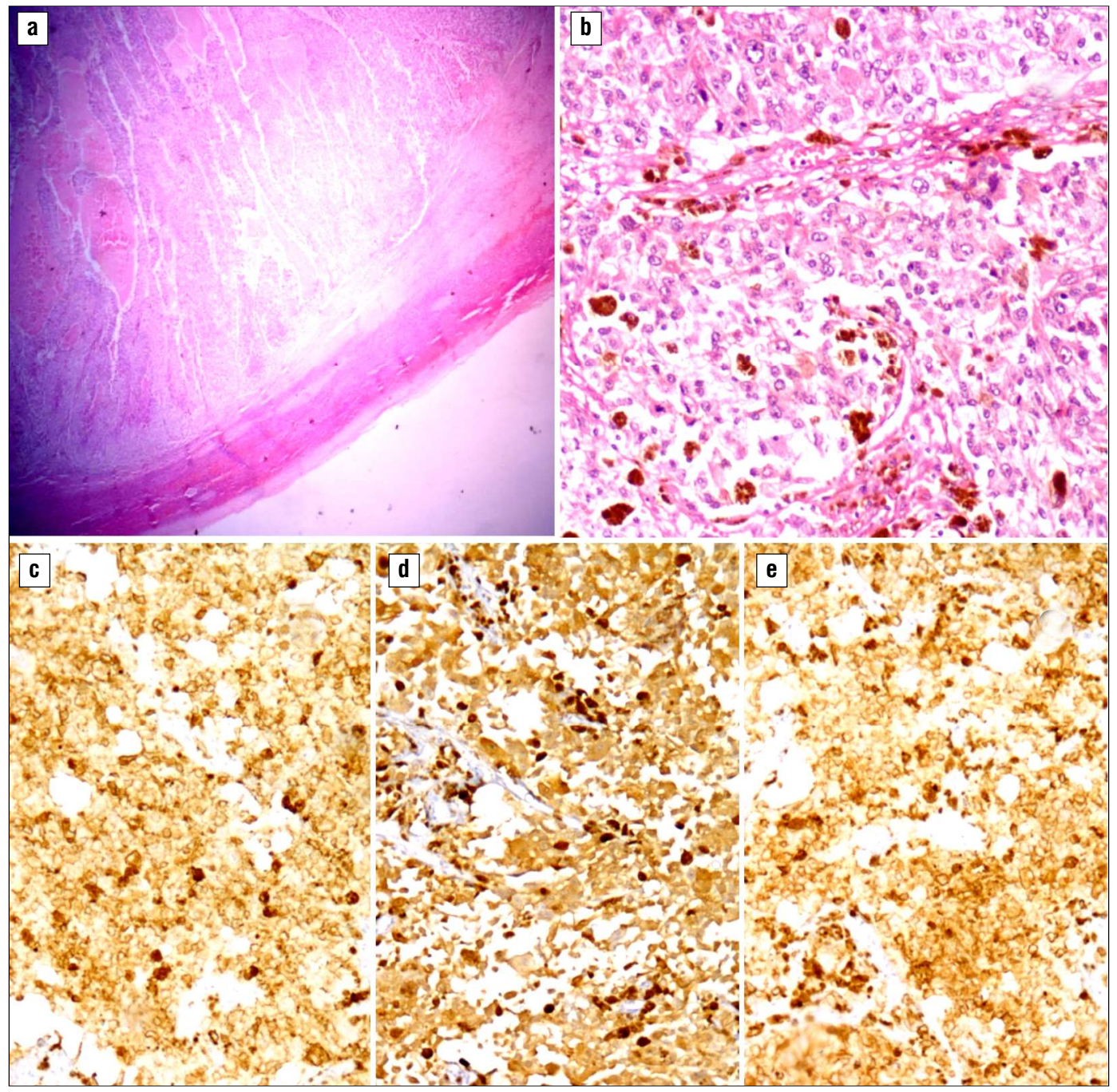

Figure 2 - Microscopic examination of the splenectomy specimen:

(a): Splenic focal lesion. Infiltration of the spleen by sheets and groups of malignant tumoral proliferation. Necrotic areas are detected as well. H\&E x40; (b): Splenic focal lesion. The neoplastic cells are pleomorphic, round to spindle and show high nucleo-cytoplasmic ratio, vesicular nuclei and prominent nucleoli. Areas of brownish pigmentation are seen as well. H\&E X200; (c): Splenic focal lesion. Diffuse positive HMB45 in the neoplastic cells (immunoperoxidase-DAB x100); (d): Splenic focal lesion. Diffuse positive $S 100$ in the neoplastic cells (immunoperoxidase-DAB x100); (e): Splenic focal lesion. Diffuse positive Melan A in the neoplastic cells (immunoperoxidase-DAB x100)

irradiation. He started receiving Dacarbazine $375 \mathrm{mg}$ daily for five days every three weeks. The patient died after the fourth cycle after a rapid deterioration of his general condition. The time interval between first presentation and death was six months.

\section{DISCUSSION}

Splenic tumors may present as a mass or as splenomegaly. Primary tumors are rare. Diffuse large B-cell lymphoma followed by hemangiosarcoma is the most common primary type. Primary splenic tumors can be categorized into lymphoid and vascular tumors.
Lymphoid tumors include Non-Hodgkin's lymphoma, Hodgkin's disease, plasmacytoma, localized lymphoid hyperplasia, Castleman's tumor, and inflammatory pseudotumor. Vascular tumors include malignant lesions such as hemangiosarcoma - which arises from the vascular endothelial cells-, borderline lesions such as hemangiopericytoma, hemangioendothelioma and littoral cell angioma as well as benign lesions such as hemangioma - which is the most common one-, lymphangioma and hamartoma (4-8) Primary mucinous and serous cystadenocarcinoma as well as pleomorphic carcinosarcoma have been reported (9-11).

The spleen is considered to be the $10^{\text {th }}$ most 

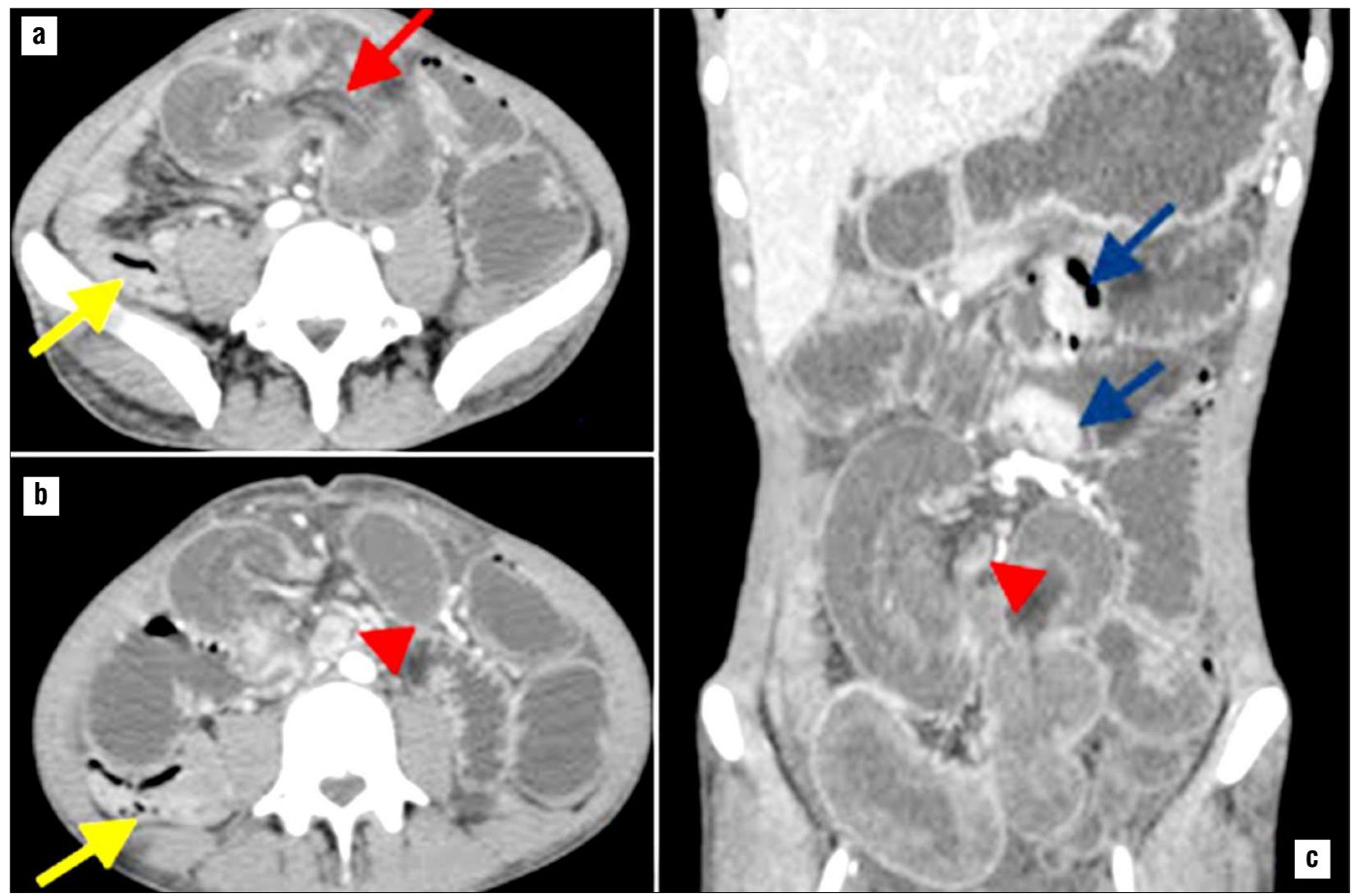

Figure 3 - Contrast-enhanced CT axial (a, b) \& coronal (c) reformatted images revealed an ileo-ileal intussusception at the right iliac region of typical bowel-within bowel configuration with invaginated mesenteric fat \& vessels (red arrow). Obstructed proximal small-bowel loops are dilated, while the colon is collapsed (yellow arrow). Few related distorted mesenteric LNs (arrowheads) are considered a causative lead point. Few enhancing serosal surface implants are seen on the wall of dilated small bowel loops (blue arrows).

frequent site of metastasis with the lung, breast, malignant melanoma and ovary being the most common primary sites for splenic metastasis. Skin melanoma carries the highest rate per primary tumor for metastasis in the spleen. Most of the splenic metastases were revealed at autopsy before the evolution of $\mathrm{CT}$ then Positron emission tomography-computed tomography (PET-CT). This rarity of incidence of metastases despite being a highly vascular organ can be attributed to many factors which are the constant blood flow, splenic contraction and lack of afferent lymphatics. The microenvironment of the spleen can have an anti-neoplastic effect as well. Determination of the lesion whether primary or secondary may be difficult in cases with no previous clear history of a skin primary $(3,6,12-14)$.

There is no typical clinical scenario for splenic tumors. Yet, abdominal left upper quadrant discomfort or pain may be present. On examination, the enlarged spleen may be palpable with normal or abnormal CBC on laboratory evaluation. In metastatic cases, symptoms and long history of treatment of the primary are usually present. Manifestations of systemic involvement such as fatigue, weight loss, anemia, thrombocytopenia, and fever may be present. Splenic rupture is rarely reported to be the initial presentation $(5,6,8$, 14). As for our patient, he was presented with left upper quadrant pain, mild splenomegaly and the splenic mass was his firstly discovered significant finding.

The US-guided biopsy from the splenic focal lesion may be sufficient for the establishment of the diagnosis, especially in case of metastatic disease and lymphoma saving the patient from the need for splenectomy (15). Yet, splenectomy still has a role in the management and is reported to offer better survival even in stage IV melanoma in selected patients as well as its role in symptoms palliation with low morbidity and mortality (12). In our patient, splenectomy was performed for reaching a final diagnosis as the preoperative biopsy was not enough for confirmation of the diagnosis and tissue biopsy was recommended.

Splenectomy can be performed either via an open or a laparoscopic approach. The open approach can be performed through a midline or a subcostal incision. The laparoscopic approach can be done either totally laparoscopic or hand-assisted. Splenectomy generally carries a low incidence of postoperative morbidity and offers sound oncologic outcomes in indicated cases $(6,7)$. In our patient, open splenectomy through midline incision was the chosen strategy for manage- 


\begin{tabular}{lll}
\hline & The presented patient & The previously reported one \\
\hline Age & 35 & 56 \\
\hline Gender & male & male \\
\hline Presentation & left upper quadrant pain & $\begin{array}{l}\text { anorexia, weight loss, and left } \\
\text { upper quadrant pain }\end{array}$ \\
\hline $\begin{array}{l}\text { Splenic focal lesion size } \\
\text { Metastasis }\end{array}$ & $10 \times 7 \times 7 \mathrm{~cm}$ & $13 \times 11 \times 15 \mathrm{~cm}$ \\
\hline Method of diagnosis & Small intestinal serosa, & Retroperitoneal lymph nodes \\
\hdashline Surgery & retroperitoneal lymph nodes & CT guided FNAC \\
\hline Chemotherapy & Yes & No \\
\hline $\begin{array}{l}\text { Time from initial diagnosis } \\
\text { to mortality }\end{array}$ & dacarbazine & cisplatin, dacarbazine, and interferon \\
\hline
\end{tabular}

ment. The patient had a smooth postoperative course with no splenectomy related complications.

Primary extra-cutaneous melanoma is rare, usually diagnosed late and carries a poorer prognosis. It constitutes about $5 \%$ of the total incidence of melanoma including uveal, genitourinary, nasopharyngeal, meningeal and gastrointestinal melanoma. Its management algorithms include surgery, immunotherapy, targeted therapy, chemotherapy and cytokine therapy $(1,16-19)$. Primary splenic melanoma is extremely rare with only one reported case in the literature (1). The differences between the previously reported patient and ours are summarized in table 1 . We considered the splenic mass as the primary because of being the lesion causing the initial presentation, being solitary lesion, the presence of nearby small intestinal secondary serosal implants as well as the absence of any clinically or radiologically detectable primary. The second possibility that may be considered is metastatic melanoma with unknown primary.

Adult intussusception is considered a rare event that accounts only for $1 \%$ of cases of adult intestinal obstruction. The majority of cases of adult intussusception are neoplastic. Melanoma is not an uncommon etiology in such a category. The patient may pass asymptomatic or he may be presented with symptoms and signs of intestinal obstruction. Resection of the involved segment is the best-suggested management approach aiming at least for symptomatic relief \& improvement of the quality of life $(20,21)$.

This report is not only unique because of the rarity of the primary splenic melanoma but the intussusception caused by melanomatous serosal deposits is extremely rare as well.

\section{CONCLUSIONS}

Melanoma is a highly aggressive tumor that carries a poor prognosis. The incidence of involvement of the spleen by melanoma is a rare event and primary splenic melanoma is much rarer. Meanwhile, small intestinal intussusception on top of primary mucosal or secondary serosal melanoma is very rare as well. Surgical intervention in symptomatic patients is the best-suggested treatment approach.

Conflict of interest: None declared

\section{REFERENCES}

1. Škedelj J, Žagar I, Snoj M. Melanoma and isolated splenic metastases - case report and literature review. Slov Med Journal. 2015; 84(6):495-500.

2. Mirfazaelian H, Oryan A, Davari A, Daneshbod K, Daneshbod Y. Spontaneous splenic rupture in melanoma. Case Rep Pathol. 2014;2014:865453.

3. Basu S, Jambhekar N. Primary malignant melanoma presenting as FDG avid large necrotic splenic mass with metastatic retroperitoneal adenopathy: FDG-PET and histopathological correlation. J Cancer Res Ther. 2011;7(1):96-7.

4. Kaza RK, Azar S, Al-Hawary MM, Francis IR. Primary and secondary neoplasms of the spleen. Cancer Imaging. 2010;10(1):173-82.

5. Fotiadis C, Georgopoulos I, Stoidis C, Patapis P. Primary tumors of the spleen. Int J Biomed Sci. 2009;5(2):85-91.

6. Silver DS, Pointer Jr. DT, Slakey DP. Solid Tumors of the Spleen: Evaluation and Management. J Am Coll Surg. 2017;224(6): 1104-11.

7. Pointer DT, Slakey DP. Cysts and Tumors of the Spleen. In: Yeo 2 Volume Set (Eighth Edition) CJBT-SS of the AT, editor. Philadelphia: Content Repository Only!; 2019. p. 1654-9.

8. Cao L, Hong J, Wang Y, Yu J, Ma R, Li J, et al. A primary splenic angiosarcoma hepatic metastasis after splenectomy and its genomic alteration profile. Medicine (Baltimore). 2019;98(28): e16245-e16245.

9. Raul SK, Yadav B, Sharma R, Joseph M. Primary Mucinous Cystadenocarcinoma of Spleen with Pseudomyxoma Peritonei: 
a Very Rare Entity. Indian J Surg. 2020;82:433-435. Epub 2019 Aug 23.

10. Ramson DM, Ackermann T, Thompson M, Gribbin J. Primary pleomorphic carcinosarcoma of the spleen: case report and review of the literature. ANZ J Surg. 2020;90(6):1209-1211. Epub 2019 Oct 31.

11. Chou CK, Chou S-C. Serous cystadenocarcinoma of the spleen. Radiol Case Reports. 2017;12(2):273-7.

12. de Wilt JHW, McCarthy WH, Thompson JF. Surgical treatment of splenic metastases in patients with melanoma. J Am Coll Surg. 2018;197(1):38-43.

13. Gavriilidis P, Goupou E. Solitary metachronous splenic metastasis from cutaneous melanoma. BMJ Case Rep. 2012;2012: bcr2012007317.

14. Silver DS, Pointer Jr. DT, Slakey DP. Solid Tumors of the Spleen: Evaluation and Management. J Am Coll Surg. 2018;224(6): 1104-11.

15. Olson MC, Atwell TD, Harmsen WS, Konrad A, King RL, Lin Y, et al. Safety and Accuracy of Percutaneous Image-Guided Core Biopsy of the Spleen. Am J Roentgenol. 2016;206(3):655-9.

16. Scott JF, Gerstenblith MR, editors. Noncutaneous Melanoma. Brisbane (AU): Codon Publications; 2018

17. Del Prete V, Chaloupka K, Holzmann D, Fink D, Levesque M, Dummer R, et al. Noncutaneous Melanomas: A Single-Center Analysis. Dermatology. 2016;232(1):22-9.

18. Wong VK, Lubner MG, Menias CO, Mellnick VM, Kennedy TA, Bhalla $\mathrm{S}$, et al. Clinical and Imaging Features of Noncutaneous Melanoma. Am J Roentgenol. 2017;208(5):942-59.

19. Kibbi N, Kluger H, Choi JN. Melanoma: Clinical Presentations Melanoma. In: Kaufman HL, Mehnert JM, editors. Cham: Springer International Publishing; 2016. p. 107-29.

20. Hong KD, Kim J, Ji W, Wexner SD. Adult intussusception: a systematic review and meta-analysis. Tech Coloproctol. 2019;23(4): $315-24$.

21. Perez MC, Sun J, Farley C, Han D, Sun AH, Narayan D, et al. Management of intussusception in patients with melanoma. J Surg Oncol. 2019;119(7):897-902. 